\title{
Atuação da fisioterapia na incontinência urinária em mulheres praticantes de atividade física: revisão bibliográfica
}

\author{
Role of physiotherapy in urinary incontinence in women who practice physical activity: Literature
} Review

\author{
Papel de la fisioterapia en la incontinencia urinaria en mujeres que practican actividad física: \\ revisión de la literatura
}

Recebido: 08/09/2021 | Revisado: 13/09/2021 | Aceito: 17/09/2021 | Publicado: 19/09/2021

\author{
Salete Silva Pereira \\ ORCID: https://orcid.org/0000-0002-4814-8499 \\ Instituto Educacional Santa Catarina-Faculdade Guaraí, Brasil \\ E-mail: salete.ssp@gmail.com \\ Brenda Munike Guedes Leite \\ ORCID: https://orcid.org/0000-0002-4809-1779 \\ Instituto Educacional Santa Catarina-Faculdade Guaraí, Brasil \\ E-mail: brendamunike@@hotmail.com \\ Karla Camila Correia da Silva \\ ORCID: https://orcid.org/0000-0003-1538-7028 \\ Instituto Educacional Santa Catarina-Faculdade Guaraí, Brasil \\ E-mail:karlacamilac@yahoo.com.br
}

\begin{abstract}
Resumo
A prevalência da incontinência urinária é significativamente maior em mulheres, fisicamente ativas e atletas de alto rendimento devido ao grande esforço realizado durante o treinamento físico. O objetivo desse estudo é descrever a atuação da fisioterapia na incontinência urinária em mulheres praticantes de atividade física, demonstrando as principais técnicas de tratamento fisioterapêutico utilizados para tratar esta disfunção que interfere direta ou indiretamente na qualidade de vida de quem sofre com esta patologia. Metodologia: Trata-se de um estudo de revisão bibliográfica. Foi realizado um levantamento bibliográfico nos bancos de dados Bireme, a Base de Dados Literatura Latino-Americana e do Caribe em Ciências da Saúde (LILACS), nas bases de dados Scientific Electronic Library Online (SciELO) e Scholar Google (Google Acadêmico). Resultados: A fisioterapia é essencial no tratamento da incontinência urinária por promover percepção corporal, devolver e melhorar a função dos músculos perineais, além de normalizar a tonicidade muscular, ajudando com que a mulher conquiste novamente uma boa continência urinária desenvolvendo assim uma melhora na sua qualidade de vida. Conclusão: Existem diversas modalidades fisioterapêuticas que podem auxiliar na melhora da função da musculatura do assoalho pélvico, entre elas, a eletroestimulação, cinesioterapia, cones vaginais, biofeedback e reeducação comportamental, contendo variados recursos e técnicas que podem ser utilizados para a melhora significativa dos sintomas da patologia e consequentemente tendo efeito positivo na qualidade de vida das pacientes. No entanto, os benefícios deste tratamento conservador se dão não somente por uma única modalidade terapêutica, mas sim pela associação das diversas formas e recursos disponíveis de tratamento mediante a queixa e sintomatologia apresentada e da boa adesão e evolução própria de cada mulher.
\end{abstract}

Palavras-chave: Incontinência urinária; Fisioterapia; Atividade física.

\begin{abstract}
The prevalence of urinary incontinence is greater in women, physically active and high-performance athletes due to the great effort performed during physical training. The aim of this study is to define the role of physical therapy in urinary incontinence in women who practice physical activity, demonstrating how the main physical therapy treatment techniques used to treat this dysfunction that directly or indirectly interferes in the quality of life of those who suffer from this pathology. Methodology: This is a literature review study, a literature review was carried out in the Bireme databases, the Latin American and Caribbean Literature Database in Health Sciences (LILACS), in the Scientific Electronic Library Online databases (SciELO) and Scholar Google (Google Scholar). Results: Physiotherapy is essential in the treatment of urinary incontinence as it promotes body awareness, restores and improves the function of the perineal muscles, in addition to normalizing muscle tone, helping women to regain good urinary continence, thus improving their quality. of life. Conclusion: there are several physiotherapeutic modalities that can help to improve the function of the pelvic outbreak muscles, including electrical stimulation, kinesiotherapy, vaginal cones, biofeedback and behavioral re-education, containing various resources and techniques that can be used to improve health levels
\end{abstract}


pathology and consequently having a positive effect on the quality of life of patients. However, the benefits of this conservative treatment are given not only by a single therapeutic modality, but by the association of different forms and resources of treatment available according to the complaint and symptoms presented and the good adherence and evolution of each woman.

Keywords: Urinary incontinence; Physiotherapy; Physical activity.

\section{Resumen}

La prevalencia de incontinencia urinaria es mayor en mujeres, deportistas físicamente activos y de alto rendimiento debido al gran esfuerzo realizado durante el entrenamiento físico. El objetivo de este estudio es definir el papel de la fisioterapia en la incontinencia urinaria en mujeres que practican actividad física, demostrando cómo las principales técnicas de tratamiento de fisioterapia emplean para tratar esta disfunción que directa o indirectamente interfiere en la calidad de vida de quienes la padecen. de esta patología. Metodología: Se trata de un estudio de revisión de la literatura, se realizó una revisión de la literatura en las bases de datos Bireme, la Base de Datos de Literatura de América Latina y el Caribe en Ciencias de la Salud (LILACS), en las bases de datos Scientific Electronic Library Online (SciELO) y Scholar Google (Google Scholar). ). Resultados: La fisioterapia es fundamental en el tratamiento de la incontinencia urinaria ya que promueve la conciencia corporal, restaura y mejora la función de la musculatura perineal, además de normalizar el tono muscular, ayudando a la mujer a recuperar una buena continencia urinaria, mejorando así su calidad de vida. Conclusión: existen varias modalidades fisioterapéuticas que pueden ayudar a mejorar la función de los músculos del brote pélvico, entre ellas la estimulación eléctrica, la kinesioterapia, los conos vaginales, la biorretroalimentación y la reeducación conductual, que contienen diversos recursos y técnicas que pueden utilizarse para mejorar los niveles de salud de la patología. y consecuentemente tener un efecto positivo en la calidad de vida de los pacientes. Sin embargo, los beneficios de este tratamiento conservador vienen dados no solo por una única modalidad terapéutica, sino por la asociación de diferentes formas y recursos de tratamiento disponibles según la queja y síntomas presentados y la buena adherencia y evolución de cada mujer.

Palabras clave: Incontinencia urinaria; Fisioterapia; Actividad física.

\section{Introdução}

A atividade física (AF) é conceituada como todo movimento corporal resultante de contração muscular com gasto de energia maior do que em repouso que, em geral, possibilita o ganho de força física, flexibilidade corporal e aumento da resistência, com alterações, seja na composição corporal ou no desempenho desportivo (Naves, Letieri, Simon, Leite, \& Letieri, 2016).

Qualidade de vida (QV) é definida como a compreensão do ser humano sobre sua situação de vida no contexto sociocultural e de valores, levando em conta seu bem-estar, estilo de vida e saúde, propósitos pessoais, hábitos e lazer. A QV abrange múltiplos aspectos próprios de cada indivíduo o que a faz ser compreendida e analisada de diferentes formas sendo que, comumente, remete a algo positivo. A atividade física e a qualidade de vida são frequentemente associadas pelo fato da prática de atividade física gerar efeitos benéficos que se relaciona com as perspectivas da qualidade de vida (Pereira, Teixeira, \& Santos, 2012; Pucci, Rech, Fermino, \& Reis, 2012).

A Sociedade Internacional de Continência define como incontinência urinária toda queixa de eliminação involuntária de urina. Caracteriza-se em três tipos mais comuns que são: Incontinência Urinária de Urgência (IUU) onde a perda urinária se associa com uma extrema e repentina vontade de urinar, Incontinência Urinária de Esforço (IUE) que é a perda de urina mediante qualquer esforço físico, seja ele mínimo como um espirro ou tosse, e, por fim, a Incontinência Urinária Mista (IUM) em que há associação de sintomas da incontinência urinária de urgência e a incontinência urinária de esforço (Melo \& Cirqueira, 2018).

A fisioterapia é essencial no tratamento da incontinência urinária por promover percepção corporal, devolver e melhorar a função dos músculos perineais, além de normalizar a tonicidade muscular, ajudando com que a mulher conquiste novamente uma boa continência urinária desenvolvendo assim uma melhora na sua qualidade de vida. Os protocolos de tratamento são capazes de atingir mulheres de diferentes idades, respeitando sempre a particularidade de cada uma na busca de resultados positivos e satisfatórios a disfunção (Henkes, Fiori, Carvalho, Tavares, \& Frare, 2015). 
Dito isso, este estudo tem como objetivo descrever a atuação da fisioterapia na incontinência urinária em mulheres praticantes de atividade física, demonstrando as principais técnicas de tratamento fisioterapêutico utilizados para tratar esta disfunção que interfere direta ou indiretamente a qualidade de vida de quem sofre com esta patologia.

\section{Metodologia}

No intuito de cumprir os objetivos, foi realizada uma pesquisa bibliográfica com a finalidade de contribuir e compartilhar conhecimentos sobre a incontinência urinária feminina e a atuação do profissional fisioterapeuta nesta disfunção.

A pesquisa bibliográfica por via de referências teóricas publicadas em livros, artigos, dissertações e/ou teses, procura a justificativa de um problema, buscando também identificar e avaliar artigos e publicações de certo tema, problema ou assunto (Bervian \& Silva, 2006). Este trabalho é de caráter qualitativo, ou seja, compreendendo diversos métodos interpretativos que se destinam a detalhar e a decifrar os elementos de um sistema complicado de significados.

Esta pesquisa é fundamentada no referencial bibliográfico, onde a literatura científica utilizada tem origem no idioma da língua portuguesa, inglesa e espanhola, considerando artigos, livros e dissertações publicados nos últimos vinte anos a fim de abranger uma maior extensão bibliográfica.

Os artigos foram selecionados a partir das seguintes palavras-chave indexadas nos Descritores em Ciências da Saúde (DeCS): "incontinência urinária feminina", "assoalho pélvico", "fisioterapia" e "qualidade de vida", sendo utilizadas estas combinações com os recursos disponibilizados pelos operadores booleanos AND e OR.

Os critérios de inclusão foram referências teóricas que tivessem como objetivo a fisioterapia no tratamento da incontinência urinária feminina, atividade física e qualidade de vida; atividade física e incontinência urinária; fisiopatologia da incontinência urinária; além de conter em seu texto informações quantitativas sobre prevalência ou incidência de incontinência urinária, os fatores de risco e/ou suas circunstâncias e de exclusão estudos repetitivos, e que não se enquadraram nos critérios de inclusão.

Para que o trabalho apresente consistência teórica, foram utilizadas as Plataformas de Pesquisa Bireme, a Base de Dados Literatura Latino-Americana e do Caribe em Ciências da Saúde (LILACS), nas bases de dados Scientific Electronic Library Online (SciELO) e Scholar Google (Google Acadêmico).

\section{Resultados e Discussão}

\subsection{A importância das academias na atividade física}

A população está cada vez mais se preocupando com a melhoria da qualidade de vida, proporcionando um grande aumento de público nas academias. A prática regular de exercícios físicos traz inúmeros benefícios à saúde e a qualidade de vida. As academias se tornaram uma opção para a população urbana, que adere ao exercício físico com o intuito de obter melhorias em seu bem-estar geral. (Filho, Jesus, \& Araújo, 2012; Moraes, 2015).

Segundo Liz e Andrade (2016), os motivos pelos quais o público procura a academia para a prática de atividade física se relacionam com a promoção de bem-estar e qualidade de vida, melhora da saúde e a preocupação com a estética corporal, além da socialização entre os praticantes no ambiente.

A musculação é a variedade de modalidade do exercício físico mais procurada e realizada nas academias. Seus efeitos benéficos dependem do bom planejamento e prática regular, assim como também de uma rotina, gerando resultados mais rápidos como, por exemplo, ganhos de massa magra, perca de gordura e também aumento da força muscular e condicionamento físico. (Liz \& Andrade, 2016).

Atualmente é cada vez mais exposto que a insatisfação corporal é uma queixa de ambos os sexos, os levando a procura de um padrão corporal estabelecido culturalmente para fazer parte do perfil estético mais desejável possível, fazendo com que 
as pessoas utilizem os recursos de atividade física da academia de forma exagerada para atingir suas perspectivas. (Alves, Pinto, Alves, Mota \& Leirós, 2009).

\subsection{Incontinência Urinária}

É de extrema importância o conhecimento sobre as estruturas da pelve, dos órgãos e dos músculos do assoalho pélvico para facilitar o entendimento sobre a ocorrência da incontinência urinária. A fusão dos ossos ílio, ísquio e púbis formam um grande osso chamado pelve que se divide em direita e esquerda. A pelve se articula com o sacro e a união da pelve direita e esquerda se dá pela sínfise púbica. Nas mulheres, esta estrutura tem abertura superior oval e arredondada, possuindo também ângulo e arco púbico largo. (Moore, Agur, \& Dalley, 2017).

A bexiga é um órgão que está localizada atrás da sínfise púbica sendo mantida nesta posição pelos ligamentos pubovesicais, ligamento fibroareolares e ligamento umbilical médio. Sua inervação é feita por nervos aferentes da medula espinhal entre o nível de S2 e S4, contento também inervação simpática. A bexiga contém elasticidade e é formada por musculatura lisa. O musculo detrusor se contrai durante a micção e as fibras musculares deste musculo são posicionadas de forma que no momento da contração da bexiga, seja capaz de ter uma contração simétrica. (Polden \& Mantle, 2002).

A micção se dá por meio de contrações musculares voluntárias e involuntárias a partir do momento em que a quantidade de urina na bexiga chega a um valor aproximado de 200 a $400 \mathrm{ml}$ resultando assim em aumento da pressão intravesical fazendo com que os receptores de estiramento da parede interna da bexiga transmitem impulsos nervosos até a medula espinal a nível sacral de S2 e S3 provocando o reflexo de micção. Esses impulsos nervosos se propagam na parede da bexiga a partir da decisão do indivíduo de urinar, e no músculo esfíncter interno da uretra levando-o ao relaxamento e a contração do músculo detrusor da bexiga. (Tortora \& Derrickson, 2016).

Santos, Vinha e Borges (2020) descreveram em seu artigo que a continência tem como conceito a capacidade normal de autocontrole urinário e fecal de um indivíduo, além de ter entendimento do local e horário apropriado para tal fim. Para isto é necessária uma boa condição dos órgãos, da musculatura e ligamentos, como também da capacidade física e saúde mental. Em adultos a micção pode haver variações, está relacionada ao volume de urina e a regularidade tanto fecal como miccional.

Baracho, Rossi e Lopes (2018), afirmam que o assoalho pélvico é compreendido como uma rede de músculos, fáscias e órgãos (útero, bexiga e ânus), que dentre as variadas funções está a de sustentar os órgãos pélvicos e a função esfincteriana anal e uretral. Os músculos superficiais e profundos que compõem o assoalho pélvico são: isquiocavernoso, transverso superficial do períneo, bulbocavernoso e esfíncter do ânus, além também do pubococcígeo, puborretal, iliococcígeo, transverso profundo do períneo, e esfíncter interno da uretra. Quaisquer alterações nas funções dessas estruturas podem levar ao desenvolvimento da incontinência urinária.

Sempre que houver a pressão intravesical na bexiga inferior e a pressão do fechamento da uretra, a continência urinária estará preservada. $\mathrm{O}$ assoalho pélvico também comunica a pressão para a bexiga e para a uretra, gerando maior grau de pressão uretral que a vesical, sendo capaz de auxiliar na mecânica da continência urinária. (Santos, Vinha \& Borges, 2020).

A Sociedade Internacional de Continência define incontinência urinária como uma perda involuntária de urina pela uretra, objetivamente demonstrável, que causa problema social e higiênico (Berquó, Ribeiro \& Amaral, 2009). É um problema considerado comum, onde mulheres de diversas idades podem ser afetadas. (Herrmann et al., 2003; Oliveira, Rodrigues \& Paula, 2007).

Segundo Caetano, Tavares e Lopes (2007), a prevalência da incontinência urinária é significativamente maior em mulheres, fisicamente ativas e atletas de alto rendimento, devido ao grande esforço realizado durante o treinamento físico. As atividades que podem causar perda de urina incluem principalmente saltos, aterrissagens de alto impacto, corrida e pegar peso (Guarisi et al., 2001). 
Outro fator comum que predispõe para a incontinência urinária, prejudicando os músculos responsáveis pela continência na mulher, é o período gestacional e durante o parto (Caetano, Tavares \& Lopes, 2007). De acordo com Carreño et al. (2015), Herrmann et al. (2003) e Oliveira, Rodrigues e Paula (2007), a incontinência urinária traz implicações sociais sendo capaz de levar a perda de autoconfiança, redução da autoestima, isolamento social, frustrações psicossociais, atingindo as questões sexuais e intervindo na qualidade de vida.

Moreno (2009) destaca que o mais frequente é a perda de urina pela uretra, quando a pressão vesical ultrapassa a uretral, na falta da atividade do músculo detrusor acompanhado do esforço físico. A perda urinária acontece em casos nas quais possui aumento da pressão intra-abdominal, como no exercício físico, tosse ou espirro. Geralmente a paciente tem um número normal de micção e não perde urina enquanto dorme, somente apenas em esforço físico (Gomes \& Silva, 2010; Nunes, 2010).

Atualmente existem diversas modalidades fisioterapêuticas que podem auxiliar na melhora da função da musculatura do assoalho pélvico, entre elas, a eletroestimulação, cinesioterapia, cones vaginais, biofeedback e reeducação comportamental (Pereira, Escobar, \& Driuso, 2012).

\subsection{Tratamento da incontinência urinária de esforço e a importância do fisioterapeuta}

A Incontinência urinária é uma fisiopatologia complexa, existindo diversas formas de tratamento, sendo eles, medicamentoso, cirúrgico e o conservador. $\mathrm{O}$ tratamento medicamentoso tem ação adrenérgica com a finalidade de aumentar o tônus da musculatura lisa uretral, podendo causar o fechamento uretral. Já no tratamento cirúrgico, o uso de slings sintéticos de uretra são os procedimentos e possibilidades de opção para o tratamento da incontinência urinária de esforço, que podem ser inseridas pela via retro púbica ou transobturadora (Rios \& Gomes, 2010).

O art. $1^{\circ}$ da resolução n .372 , de 6 novembro de 2009 dispõe "Reconhecer a Fisioterapia na Saúde da Mulher como especialidade própria do profissional Fisioterapeuta.” (COFFITO). De acordo com Silva, Freitas, Scapelini e Haddad (2014), os profissionais de fisioterapia do assoalho pélvico possuem um recurso considerado efetivo e bem requisitado que é o tratamento conservador, mediante diversas técnicas, dando motivação ao paciente, constituindo assim uma importante parte para um tratamento satisfatório.

Deste modo, a fisioterapia do assoalho pélvico tem o intuito de dispor-se a evolução da musculatura do mesmo, realizando diversas técnicas, dentre elas estão o biofeedback, cones vaginais, cinesioterapia, eletroestimulação e reeducação comportamental, sendo este através de artifícios como o diário miccional. (Reis; Filho; Júnior, 2010; Silva et al., 2010).

A terapia comportamental é, geralmente, a primeira escolha para iniciar o tratamento, começando pela investigação das alterações relacionadas à sintomatologia e meio social para assim alterar hábitos miccionais ruins da paciente. Esta terapia auxilia no entendimento da reeducação da prática urinária, orientando sobre métodos para melhorar o controle e a vontade de urinar, além também de alertar sobre o regime de ingestão hídrica juntamente com o trabalho de exercícios para fortalecimento dos MAP e de consciência corporal. (Pereira, Camac, Mesquita, \& Costa, 2019).

Nos achados de Pereira, Escobar e Driusso (2012) os autores ressaltam a perspectiva de que o fortalecimento do assoalho pélvico é o procedimento mais apontado como eficaz. A terapêutica conservadora proporciona à mulher uma abordagem menos invasiva, não onerosa, aliviando o vasto desconforto. Porém, grande parte do sucesso depende da motivação e do comprometimento tanto da paciente como da equipe multidisciplinar. (Herrmann et al., 2003).

Os procedimentos mais conhecidos para a prevenção e tratamento da incontinência urinária de esforço, são os que foram criados e difundidos pelo Dr. Arnold Kegel, na década de 50. O ginecologista norte-americano usou a cinesioterapia pela primeira vez na atividade de fortalecimento dos músculos do assoalho pélvico, tendo como objetivo melhora da força e função da musculatura, favorecendo o mecanismo de continência urinária. (Caetano et al. 2007; Silva et al. 2010).

Segundo Rodrigues (2008) o recurso cinesioterapia é uma técnica bastante utilizada no tratamento fisioterápico para o 
fortalecimento da MAP, ela trabalha os músculos pelo movimento, podendo ser utilizada de duas formas: ativa e passiva. Na forma ativa, o paciente realiza sozinho o movimento de forma voluntária. Na forma passiva, os movimentos são realizados pelo fisioterapeuta através de recursos com aparelhos específicos, que substituem os exercícios físicos passivos, executando diferentes segmentos com o auxílio de diversas metodologias especiais (Rett, Simões, Herrmann, Gurgel, \& Morais, 2007).

Oliveira et al. (2007) descreveram um programa exercícios para o fortalecimento do MAP, recomendados por Kegel, que foi o primeiro a descrever de modo sistêmico, este método de tratamento conservador. Kegel, ao desenvolver este método, sustentou se na tese, que a MAP era responsiva a medidas que promovessem sua contração. A partir daí, aplicou este programa clinicamente por muitos anos, que a chamou de terapia fisiológica. Esta consistia em exercícios para o fortalecimento dessa cadeia muscular.

Os exercícios da série de Kegel baseiam-se em contrações voluntárias da musculatura do assoalho pélvico (Figura 1), onde intercala as contrações mantidas com as rápidas com o propósito de estimular as fibras musculares do tipo I e do tipo II do assoalho pélvico. O posicionamento em decúbito dorsal e ventral promovem maior facilidade e eficiência na contração do períneo (Guerra, Rossato, Nunes, \& Latorre 2014; Silva et al. 2010).

Figura 1 - Exercícios de Kegel.

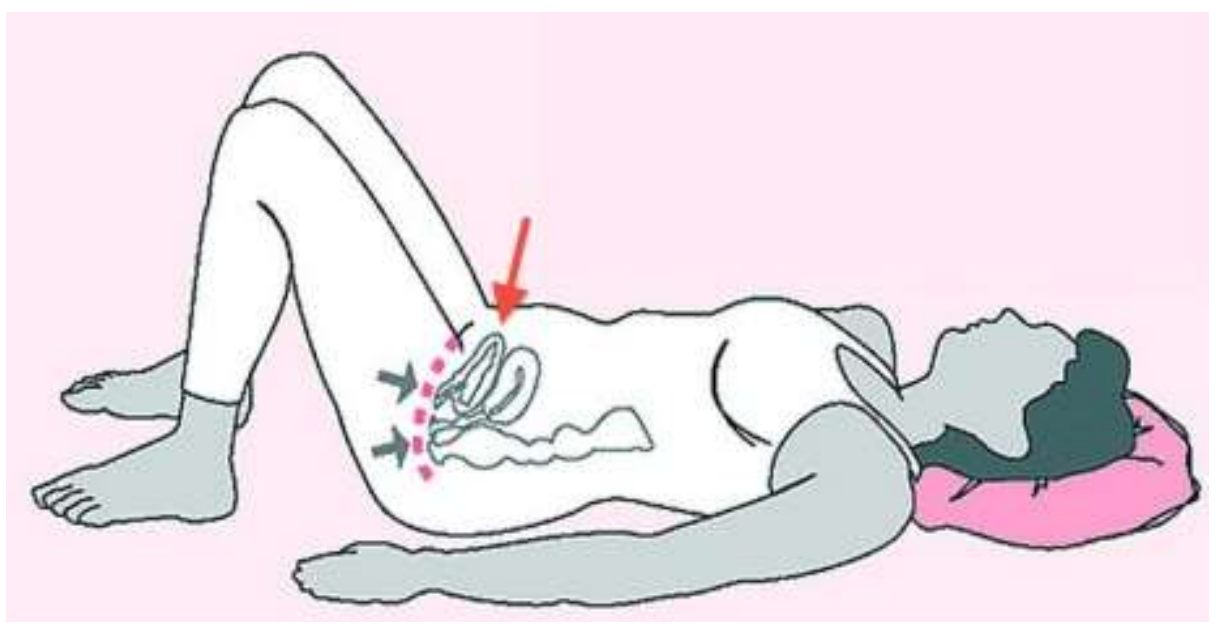

Fonte: http://pilateseciencia.blogspot.com.br/.

Outros recursos que podem ser utilizados em conjunto com a cinesioterapia são os cones vaginais, que é uma técnica intracavitária, constituído por um conjunto de cinco pesos diferentes, variando aproximadamente de 20 a 70 gramas (Figura 2). Os cones vaginais promovem um ganho de força e resistência muscular por meio do recrutamento da musculatura pubococcígea auxiliar periférica e maior conscientização perineal (Polden \& Mantle, 2002; Seleme, 2002).

Os exercícios utilizando os cones vaginais são uma intervenção de treinamento de resistência onde os mesmos são colocados dentro da vagina da mulher, sendo primeiramente posto um preservativo antes da sua inserção no canal vaginal, começando na posição onde há ajuda da gravidade, evoluindo assim para posições contra a gravidade. A promoção de um feedback proprioceptivo por meio desta técnica é capaz de produzir uma boa e melhor percepção do controle urinário (Stephenson \& O'Connor, 2004). 
Figura 2 - Técnica intracavitária utilizando cones vaginais.

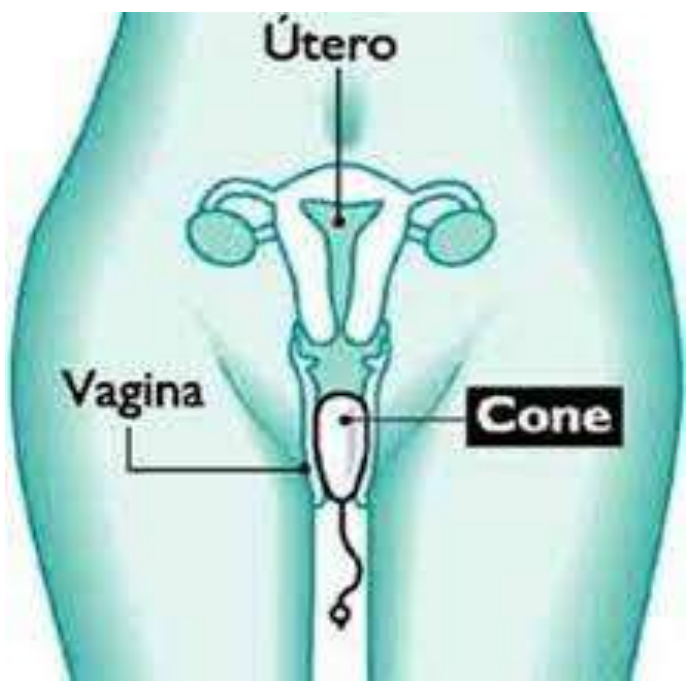

Fonte: http://espacointimofisioterapia.com.br/exercitadores-pelvicos-cones-vaginais/.

Outra modalidade é o Biofeedback (BF), um aparelho que registra as contrações musculares do assoalho pélvico e traduz sua intensidade por sinais visuais (Figura 3). É introduzida na vagina uma sonda inflável, depois de insuflada a paciente realiza uma contração máxima, sendo visualizada e graduada no visor do aparelho em mmHg. O objetivo do tratamento por BF é de ajudar as pacientes a desenvolver maior percepção e controle voluntário dos músculos do assoalho pélvico (Moreno, 2004). Segundo Capelini et al. (2006), o biofeedback utilizando o método de eletromiografia de superfície (sEMG), que é a forma mais comum de aplicação, é realizado a partir de uma sonda vaginal que possibilita ao fisioterapeuta interpretar a atividade elétrica dos músculos do assoalho pélvico e usitar essas informações para elaborar jogos, programas e exercícios conforme o grau de evolução da paciente. Com o uso deste tipo de BF, as pacientes conseguem reconhecer acertadamente a musculatura em contração e a atividade do mesmo dependendo do exercício proposto, que normalmente é representada por uma animação ou por um jogo na tela. 
Figura 3 - Biofeedback (BF).

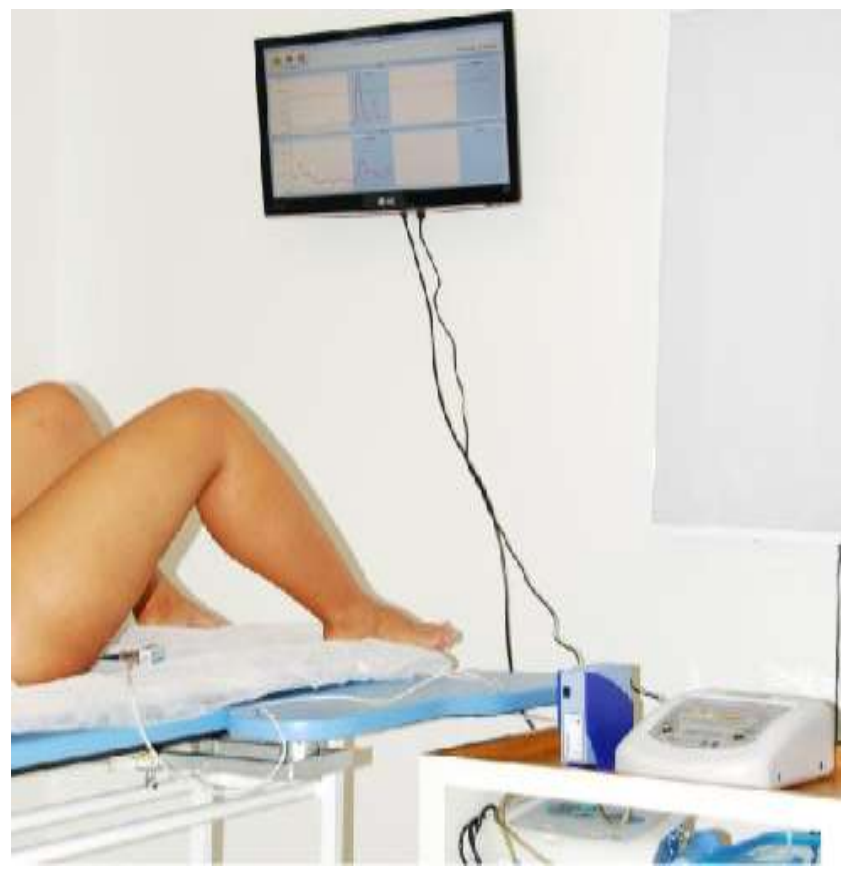

Fonte: https://www.fef.br/upload_arquivos/geral/arq_5950f2933dbcf.pdf

Há também a eletroestimulação, que consiste na colocação intravaginal de um dispositivo aproximadamente $7 \mathrm{~cm}$ de comprimento e 2,5 de diâmetro. Esta corrente consiste em um estímulo elétrico de baixa frequência de 10 e $50 \mathrm{~Hz}$, onde sua intensidade é ajustada a nível da paciente, para não se torne desagradável, sendo suficiente para que seja percebida a contração da musculatura pélvica. A técnica envolve a estimulação do nervo pudendo, realizando a contração passiva da musculatura perineal, sendo muito eficaz na conscientização do assoalho pélvico e reforço muscular (Seleme, 2002; Silva, 2003) (Figura 4).

Quanto à forma de aplicação da estimulação elétrica varia, podendo ser usado eletrodos de superfície ou eletrodos vaginais e/ou anais, sendo os últimos os mais indicados. A frequência da corrente mais frequentemente indicada é a de 50Hz por permitir a estimulação da contração das unidades motoras todas ao mesmo tempo. A largura de pulso geralmente varia de 0,21ms, sendo a amplitude de 0-100mA. Quanto ao tempo de passagem da corrente e o tempo de descanso pode estar 1:1 ou 1:2. O tempo de aplicação é em torno de 15 a 30 minutos diários podendo ser intercalados durante a semana também. A eletroestimulação gera efeitos benéficos ao tratamento da incontinência urinária, porém seus resultados são melhores quando associados a outros métodos de tratamento (Cestári, Souza, \& Silva, 2016).

Figura 4 - Eletroestimulação elétrica.

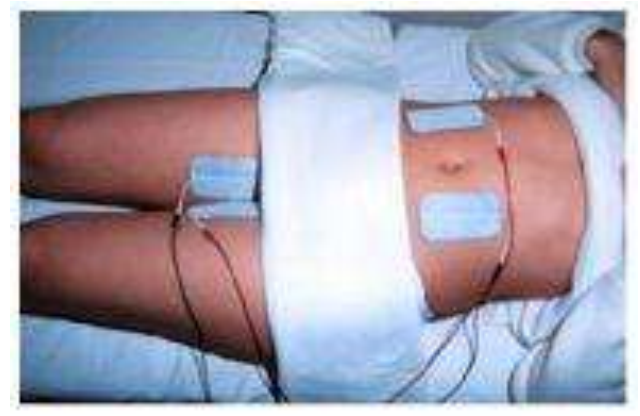

Fonte: http://www.fisiobemviver.com.br/admin/auxi/FCKeditor/image/eletroterapia/abdominal.jpg 


\section{Considerações Finais}

Conforme mencionado acima, a fisioterapia é de suma importância no tratamento da incontinência urinária feminina, contendo variados recursos e técnicas, entre elas: Terapia comportamental, Cinesioterapia, Cones Vaginais, Biofeedback e a eletroestimulação que podem e são utilizados para a melhora significativa dos sintomas da patologia e consequentemente tendo efeito positivo na qualidade de vida das pacientes. No entanto, os benefícios deste tratamento conservador se dão não somente por uma única modalidade terapêutica, mas sim pela associação das diversas formas e recursos disponíveis de tratamento mediante a queixa e sintomatologia apresentada e da boa adesão e evolução própria de cada mulher. Dito isso, conclui-se que a fisioterapia, evidenciada pela literatura, é um elemento eficaz e imprescindível na IU, porém se faz necessário mais estudos na área para confirmar a confiabilidade e aperfeiçoar o sucesso desta terapia.

\section{Referências}

Alves, D., Pinto, M., Alves, S., Mota, A., \& Leirós, V. (2009). Cultura e imagem corporal. Revista Motricidade, 5, 1-20. https://doi.org/10.6063/motri cidade.5(1). 184

Baracho, E., Rossi, L., \& Lopes, G. (2018). Fisioterapia aplicada à saúde da mulher (6a ed.). Guanabara Koogan.

Berquó, M. S., Amaral, W. N., \& Filho, J. R. (2013). Fisioterapia no tratamento da urgência miccional feminina. Revista Femina, 41(2), 107-112. http://bases.bireme.br/cgi-bin/wxislind.exe/iah/online/?IsisScript=iah/iah.xis\&src=google\&base=LILACS\&Iang=p\&nextAction=Ink\&exprSearch=69 4484\&indexSearch=ID.

Berquó, M. S., Ribeiro, M. O., \& Amaral, R. G. (2009). Fisioterapia no tratamento da incontinência urinária feminina. Revista Femina, 37(7), 385-388. http://www.febrasgo.org.br/site/wpcontent/uploads/2013/05/Feminav37n7p385-8.pdf.

Caetano, A. S., Tavares, M. C., \& Lopes, M. H. (2007). Incontinência urinária e a prática de atividades físicas. Revista Brasileira de Medicina do Esporte, 13(4), 270-274. https://www.scielo.br/j/rbme/a/99869n8hHmzgdB4sK59RWFd/?lang=pt.

Capelini, M.V., Riccetto, C. L., Dambros, M., Tamanini, J. T., Herrmann, V., \& Muller, V. (2006). Pelvic floor exercises with biofeedback for stress urinary incontinence. International braz j urol. 32(4), 462-469. https://www.scielo.br/j/ibju/a/hgBpkYrP9QcLkCyqkXNykrR/?lang=en\#.

Carreño, L. M., Angarita-Fonseca, A., Pinto, A. L., Delgado, A. N., \& García, L. M. (2015). Calidad de vida relacionada con salud e incontinencia urinaria en mujeres con exceso de peso de Bucaramanga, Colombia. Revista Ciencias de la Salud,13(1), 63-76. http://www.scielo.org.co/scielo.php?sc ript=sci_arttext\&pid=S1692-72732015000100006\&Ing=en\&nrm=iso.

Cestári, C. E., Souza, T. H., \& Silva, A. S. (2016). Eletroestimulação no tratamento da incontinência urinária de esforço feminina. Revista Ciência e Estudos Acadêmicos de Medicina, 93-101. https://periodicos.unemat.br/index.php/revistamedicina/article/view/1771

COFFITO. Conselho Federal De Fisioterapia E Terapia Ocupacional. Resolução n 372 de 6 de novembro de 2009. https://www.coffito.gov.br/nsite/?p=3135. Acesso em: 19 Maio 2021.

Filho, I. B., Jesus, L. L., \& Araújo, L. G. (2012). Atividade física e seus benefícios à saúde (Trabalho de Conclusão de Curso). Licenciatura em Educação Física, Faculdade de Educação e Meio Ambiente (FAEMA), Ariquemes, RO, Brasil. https://www.inesul.edu.br/revista/arquivos/arq-idvol_31_1412869196.pdf.

Gomes, G. V., \& da Silva, G. D. (2010). Incontinência urinária de esforço em mulheres pertencentes ao Programa de Saúde da Família de Dourados (MS). Revista da Associação Médica Brasileira, 56(6), 649-654. http://www.scielo.br/scielo.php?script=sci_arttext\&pid=S010442302010000600011\&Ing=en\&nrm=iso.

Guarisi, T., Neto, A. M., Osis, M. J., Pedro, A.O., Paiva, L. H., \& Faúndes, A. (2001). Incontinência urinária entre mulheres climatéricas brasileiras: investigação domiciliar. Revista Saúde Pública, 35(5), 428-435. http://www.scielo.br/scielo.php?script=sci_arttext\&pid=S0034-89102001000500004\&Ing=en\&nrm=iso.

Guerra, T. E., Rossato, C., Nunes, E. F., \& Latorre, G. F. (2014) Atuação da fisioterapia no tratamento de incontinência urinaria de esforço. Revista Femina, 42(6), 251-254. http://files.bvs.br/upload/S/0100-7254/2014/v42n6/a4823.pdf.

Henkes, D. F., Fiori, A., Carvalho, J. A., Tavares, K. O., \& Frare, J. C. (2015). Incontinência urinária: o impacto na vida de mulheres acometidas e o significado do tratamento fisioterapêtico. Revista Semina: Ciências Biológicas e da Saúde, 36(2), 45-56. http://www.uel.br/revistas/eu 1/index .php/seminabio/article/view/21746.

Herrmann, V., Potrick, B. A., Palmas, P. C., Zanettini, C. L., Marques, A., \& Júnior, N. R. (2003). Eletroestimulação transvaginal do assoalho pélvico no tratamento da incontinência urinária de esforço: avaliações clínica e ultrassonográfica. Revista da Associação Médica Brasileira, 49(4), 401-405. https://www.scielo.br/j/ramb/a/hdyQ7BXyrmFbDHRKCGdSNYS/?lang=pt\#. https://doi.org/10.1590/S0104-42302003000400031.

Liz, C. M., \& Andrade, A. (2016). Análise qualitativa dos motivos de adesão e desistência da musculação em academias. Revista Brasileira de Ciências do Esporte, 38(3), 267-274. http://www.scielo.br/scielo.php?script=sci_arttext\&pid=S0101-32892016000300267\&Ing=en\&nrm=iso.

Melo, A. T., \& Cirqueira, R. P. (2018). Incontinência urinária em mulheres praticantes de musculação. Id online. Revista de Psicologia, 12(42), 525-535. https://idonline.emnuvens.com.br/id/article/view/1455\#: :text=Conclui\%2Dse\%20que\%20n\%C3\%A3o\%20houve,muscula\%C3\%A7\%C3\%A3o\%20e\%20a\% 20incontin\%C3\%AAncia\%20urinaria. 
Moore, K. L., Agur, A. M., \& Dalley, A. F. (2014). Fundamentos de anatomia clínica (7a ed.). Guanabara Koogan.

Moraes, L. M. (2015). Aspectos Motivacionais para a Prática de Exercícios em Academias (Trabalho de conclusão de curso). Curso de Educação Física, Centro Universitário de Brasília - UniCEUB, Faculdade de Ciências da Educação e Saúde, Brasília, DF, Brasil. https://repositorio.uniceub. br/jspui/bitstream/235/7518/1/21237438.pdf.

Moreno, A. L. (2009). Fisioterapia em uroginecologia (2a ed.). Manole.

Naves, P. P., Letieri, R. V., Simon, I. S., Leite, S. N., \& Letieri, M. (2016). Avaliação da qualidade de vida em mulheres com sintomas de incontinência urinária de esforço praticantes de atividade física. Revista Inspirar: movimento \& saúde, 8(1), 10-16. https://inspirar.com.br/wp-content/uploads/2016/04/a rtigo2_ed37_jan -fev-mar-2016.pdf.

Nunes, Pedro. Diagnóstico de Incontinência Urinária Feminina. 2010. http://www.apnug.pt/docs/diagnostico_de_incontinencia_urinaria_feminina.pdf.

Oliveira, K. A., Rodrigues, A. B., \& Paula, A. B. (2007). Técnicas fisioterapêuticas no tratamento e prevenção da incontinência urinária de esforço na mulher. Revista Eletrônica F@pciência, 1(1),31-49. http://www.fap.com.br/fap-ciencia/edicao_2007/004.pdf.

Pereira, É. F., Teixeira, C. S., \& Santos, A. (2012). Qualidade de vida: abordagens, conceitos e avaliação. Revista Brasileira de Educação Física e Esporte, 26(2), 241-250. http://www.scielo.br/scielo.php?script=sci_arttext\&pid=S1807-55092012000200007\&lng=en\&nrm=iso. https://doi.org/10.1590/S1 807-55092012000200007.

Pereira, P. B., Camac, L. A., Mesquita, F. A., \& Costa, M. C. (2019). Incontinência urinária feminina: uma revisão bibliográfica. Revista Eletrônica Acervo Saúde, 11(14), e1343. https://acervomais.com.br/index.php/saude/article/view/1343. https://doi.org/10.25248/reas.e1343.2019.

Pereira, V. S., Escobar, A. C., \& Driusso, P. (2012). Efeitos do tratamento fisioterapêutico em mulheres idosas com incontinência urinária: uma revisão sistemática. Revista Brasileira de Fisioterapia, 16(6), 463-468. https://www.scielo.br/j/rbfis/a/m6hcBtMgSYnX8mkpQYtCjjF/?format=pdf\&lang=pt.

Polden, M., \& Mantle, J. (2002). Fisioterapia em ginecologia e obstetrícia (2a ed.). Santos.

Pucci, G. C., Rech, C. R., Fermino, R. C., \& Reis, R. S. (2012). Associação entre atividade física e qualidade de vida em adultos. Revista de Saúde Pública, 46(1), 166-179. https://www.scielo.br/scielo.php?script=sci_arttext\&pid=S0034-89102012000100021.

Reis, R. B., Filho, M. Z., \& Júnior, A. N. (2010). Urologia Fundamental. Planmark. http://www.sbu-sp.org.br/admin/upload/os1688-completourologiafundamental-09-09-10.pdf.

Rett, M. T., Simões, J. A., Herrmann, V., Gurgel, M. S., \& Morais. S. S. (2007). Qualidade de vida em mulheres após tratamento da incontinência urinária de esforço com fisioterapia. Revista Brasileira de Ginecologia e Obstetrícia, 29(3), 134-140. https://www.scielo.br/j/rbgo/a/HLzn xCKmzBGLXMYLsw5JWxG/?lang=pt\#.

Rios, L. A., \& Gomes, H. P. (2010). Incontinência urinária de esforço. In: A. Nardozza, M. Zerati, \& R.B. Reis. Urologia fundamental (Cap.29, pp. 260-263). São Paulo: Planmark.

Rodrigues, B. P. (2008). Abordagem Fisioterapêutica na Incontinência Urinária de Esforço na mulher idosa (Trabalho de conclusão de curso). Curso de Fisioterapia, Universidade Veiga de Almeida, Rio de Janeiro, RJ, Brasil. https://www.uva.edu.br/sites/all/themes/uva/files /pdf/abordagem_fisioterapeutica_incontinenci a_urinaria_de_e sforco_na_mulher_idosa.pdf.

Santos, I.L., Vinha, E. C., \& Borges, A. R. (2020). A representação das mulheres sobre a incontinência urinária (IU): um dos sofrimentos do gênero. Humanidades \& Tecnologia (FINOM), 20(1), 341-368. http://revistas.icesp.br/index.php/FINOM_Humanidade_Tecnologia/article/view/1026.

Seleme M. (2002). Diálogo Profissional sobre uroginecologia. Fisio \& Terapia, 33, 20-23.

Silva, G. C., Freitas, A.O., Scapelini, P., \& Haddad, C. A. (2014). Tratamento fisioterapêutico da incontinência urinária de esforço - relato de caso. Revista UNILUS Ensino e Pesquisa, 11(25), 18-26. http://revista.unilus.edu.br/index.php/ruep/article/view/203/u2014v11n25e203.

Silva, K. J. da et al. (2010). Intervenção fisioterapêutica na incontinência urinária de esforço (IUE) - estudo de caso. IV Seminário de Fisioterapia Uniamérica: Iniciação Científica.

Stephenson, R. G., \& O’Connor, L. J. (2004). Fisioterapia aplicada a ginecologia e obstetricia (2a ed.). Manole.

Tahara, A. K., Schwartz, G. M., \& Silva, K. A. (2003). Aderência e manutenção da prática de exercícios em academias. Revista Brasileira Ciência e movimento, 11(4), 7-12. https://portalrevistas.ucb.br/index.php/RBCM/article/viewFile/519/543.

Tortora, G. J., \& Derrickson, B. (2016). Princípios de anatomia e fisiologia (14a ed.). Guanabara Koogan. 